\title{
Displasia Arritmogênica do Ventrículo Direito
}

\author{
Jorge Elias, Joelci Tonet, Robert Frank, G uy Fontaine
}

Vitória, ES - Paris, França

A displasia arritmogênica do ventrículo direito (DAVD) é uma entidade clínica que, originalmente, foi identificada em um grupo de pacientes submetidos a tratamento cirúrgico de taquicardia ventricular (TV), não sensível à terapêutica antiarrítmica e sem antecedentes de doença cardíaca ${ }^{1}$. A DAVD foi, recentemente, incluída no quadro das cardiomiopatias, por se tratar de uma alteração estrutural do músculo cardíaco, caracterizada por uma substituição progressiva das células miocárdicas por tecido fibrogorduroso e de causa ainda desconhecida. Algumas formas são também identificadas, como cardiomiopatia arritmogênica do ventrículo direito(VD).

A DAVD é, geralmente, identificada em adultos jovens, com predomínio no sexo masculino. Seu extenso espectro de apresentação consiste desde extra-sistolia ventricular isolada até TV sustentada. A DAVD é, provavelmente, a causa de 5,9\% das mortes súbitas de origem cardíaca que pode se apresentar como primeira manifestação da doença ${ }^{2-5}$. A morte súbita cardíaca pode ocorrer durante a prática desportiva ou mesmo em repouso.

Estes distúrbios do ritmo têm sua origem dentro de zonas estruturalmente anormais de um VD, variavelmente dilatado, recoberto de tecido fibro-adiposo de concentração e distribuição mais importante, que costumeiramente encontrado. Estas anomalias morfológicas podem ser identificadas por ecocardiograma, ventriculografia radiosotópica, ressonância magnética (RM) nuclear ou angiografia ${ }^{6}$.

\section{Forma típica}

A forma típica da DAVDé mais comumente encontrada nos indivíduos jovens, do sexo masculino e sem antecedentes cardiovasculares. Em alguns casos, pode-se encontrar um antecedente infeccioso sugerindo uma miocardite prévia. Palpitações, tonturas e mesmo síncope são os sintomas principais da doença, que são devido à TV, e mais raramente, relacionados às extra-sístoles ventriculares. É conhecida a correlação do início da TV com esforço e, entre DAVD e morte súbita em atletas ${ }^{7-11}$ (fig. 1). Em estudo post-mortem realizado por um grupo italiano observou-se que $20 \%$ de 60 pacientes $<35$ anos com morte súbita tinham achados

Instituto de Cardiologia do Espírito Santo, Vitória e Hospital Jean Rostand, Paris Correspondência: Jorge Elias - Instituto de Cardiologia do ES - Rua Alaôr Queiroz de Araújo, 220 - 29055-010 - Vitória, ES

Recebido para publicação em 6/10/97

Aceito em 26/3/98 histopatológicos consistentes com DAVD ${ }^{12}$. Além da morte súbita em jovens e atletas sem antecedentes, deve-se também atentar para os riscos de episódios de TV rápidas, que podem ocasionar perda transitória da consciência, principalmente, em indivíduos que trabalham em profissão de risco, podendo assim, gerar conseqüências catastróficas.

Outro dado importante e contrastante é a presença freqüente do exame físico praticamente normal, quando em ritmo sinusal ${ }^{10}$. Pode-se identificar, eventualmente, $4^{\mathrm{a}}$, e mais raramente, $3^{\mathrm{a}}$ bulha à ausculta cardíaca.

A presença de antecedentes familiares apresenta uma variação significativa entre as séries publicadas ${ }^{11-14}$.

\section{Diagnóstico}

Eletrocardiograma (ECG) - Os principais sinais eletrocardiográficos em ritmo sinusal são: inversão da onda $\mathrm{T}$ nas derivações precordiais direita (54\%), duração do complexo QRS > 110ms nestas mesmas derivações. A identificação de QRS de duração $>110 \mathrm{~ms}$, em ritmo sinusal na derivação $\mathrm{V}_{1}$, em indivíduo sem doença cardíaca aparente permite identificar a doença com uma sensibilidade de 55\% e especificidade de $100 \%{ }^{15}$. As ondas épsilon decorrentes da pós-excitação ventricular (fig. 2), são observadas em $30 \%$ dos casos. Trata-se de uma deflexão de baixa amplitude, de curta duração, sucedendo imediatamente o complexo QRS, mais particularmente, visível nas derivações precordiais direitas. Pode-se lançar mão de derivações bipolares em precórdio, derivação de Lewis e correlatas, para se obter registro desse potenciais fragmentados sugestivos de retardo da ativação ventricular direita ${ }^{16}$. Os outros sinais, bloqueio de ramo direito (15\%), distúrbio de condução pelo ramo direito (18\%), complexos de baixa voltagem e hipertrofia ventricular direita são mais raros e traduzem uma forma difusa da doença com possível comprometimento do sistema de condução atrioventricular ${ }^{17}$.

OECG durante a TV mostra um aspecto de bloqueio de ramo esquerdo (BRE) sugerindo ser o sítio de origem da arritmia localizado no VD. O eixo do QRS é normal ou desviado para a direita, quando a TV se origina da via de saída do $\mathrm{VD}$, ou pode apresentar desvio esquerdo, quando originária de ápex ou parede diafragmática do VD (fig. 3). Taquiarritmias supraventriculares têm sido observadas em $24 \%$ dos $\operatorname{casos}{ }^{18}$.

Teste de esforço - OECG de esforço pode demonstrar aparecimento ou intensificação da arritmia ventricular durante o pico do esforço ou na fase de recuperação. É interes- 
sante a observação de ser mais fácil a indução de arritmias na forma localizada que na forma global da doença (65\%$29 \%$; p<0,01). Neste caso, a implicação prognóstica dos dados obtidos através do teste de esforço possa ser de uma progressão da doença mais de que uma diminuição de sua severidade ${ }^{19}$.

Holter - O registro de ECG de $24 \mathrm{~h}$ pode mostrar uma arritmia ventricular na forma de extra-sistolia ventricular isolada, acoplada, TV não sustentada e sustentada que se intensificam com esforço, com predomínio durante a vigília e, às vezes, associada a distúrbios do ritmo supraventricular.

Eletrocardiografia de alta resolução (ECGAR) - Os potenciais tardios são a tradução elétrica de uma zona de condução lenta e heterogênea, refletindo assim a presença de um substrato arritmogênico ${ }^{20}$. Na presença de condições eletrofisiológicas e autonômicas predisponentes, a arritmia por reentrada pode se mostrar presente. Existe uma tendência, em vários grupos, em considerar o ECGAR como o método mais sensível para o diagnóstico da DAVD $(85 \%)^{21,22}$. A combinação da análise pelo domínio do tempo e da freqüência melhora a sensibilidade na pesquisa para DAVD sem reduzir a especificidade do método ${ }^{23}$. Entretanto, se a doença é localizada, o ECGAR pode ser normal mesmo em pacientes com DAVD e arritmia ventricular ${ }^{24}$. Estudo com ECGAR seriado não foi capaz de predizer a suscetibilidade para TV em portadores de DAVD ${ }^{25}$.

Ecocardiograma - Os estudos ecocardiográficos evidenciam a dilatação das câmaras direitas nas formas difusas da doença. Outros achados são aumento no diâmetro sistólico e diastólico final do VD, zona discinética em parede ínfero-basal, aneurismas localizados e dilatação da via de entrada e saída ou discinesia apical e desarranjo trabecular (fig. 4). Ao contrário, nas formas ditas menores ou "frustras", os dados tornam-se de apreciação e reprodutibilidade variável na dependência da experiência do ecocardiografista. Muitos autores consideram que a ecocardiografia tem valor diagnóstico apenas nos casos com quadro clínico mais relevante ${ }^{26-30}$. Porém, Scognamiglio e col ${ }^{31}$ sugerem que o ecocardiograma pode ser útil para o diagnóstico precoce da DAVD, mesmo em pacientes assintomáticos, devido à possibilidade de identificação de alterações morfológicas e na cinética ventricular direita.

Radiografia de tórax - Este exame mostra, mais freqüentemente, uma cardiomegalia ligeira geralmente atribuída às recidivas dos distúrbios do ritmo. Geralmente, o índice cardiotorácico é <0,6. Geralmente, não existe padrão

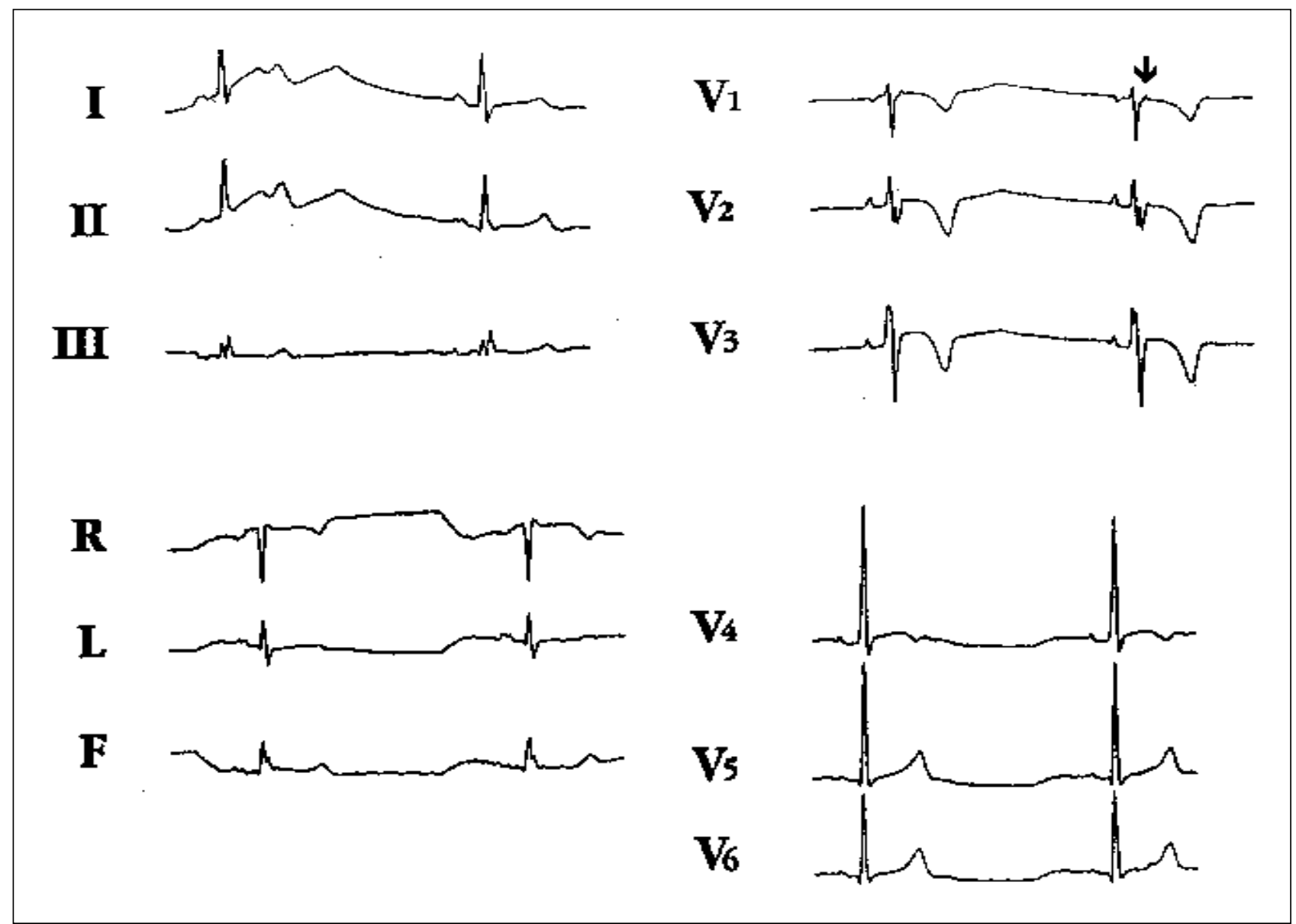

Fig. 1- Eletrocardiograma de 12 derivações de portador de DAVD. Observar a presença de inversão da onda T em precordiais direita, QRS de duração >110ms e distúrbio de condução pelo ramo direito. Presença de onda "épsilon" na porção final do QRS da derivação $\mathrm{V}_{1}$. 


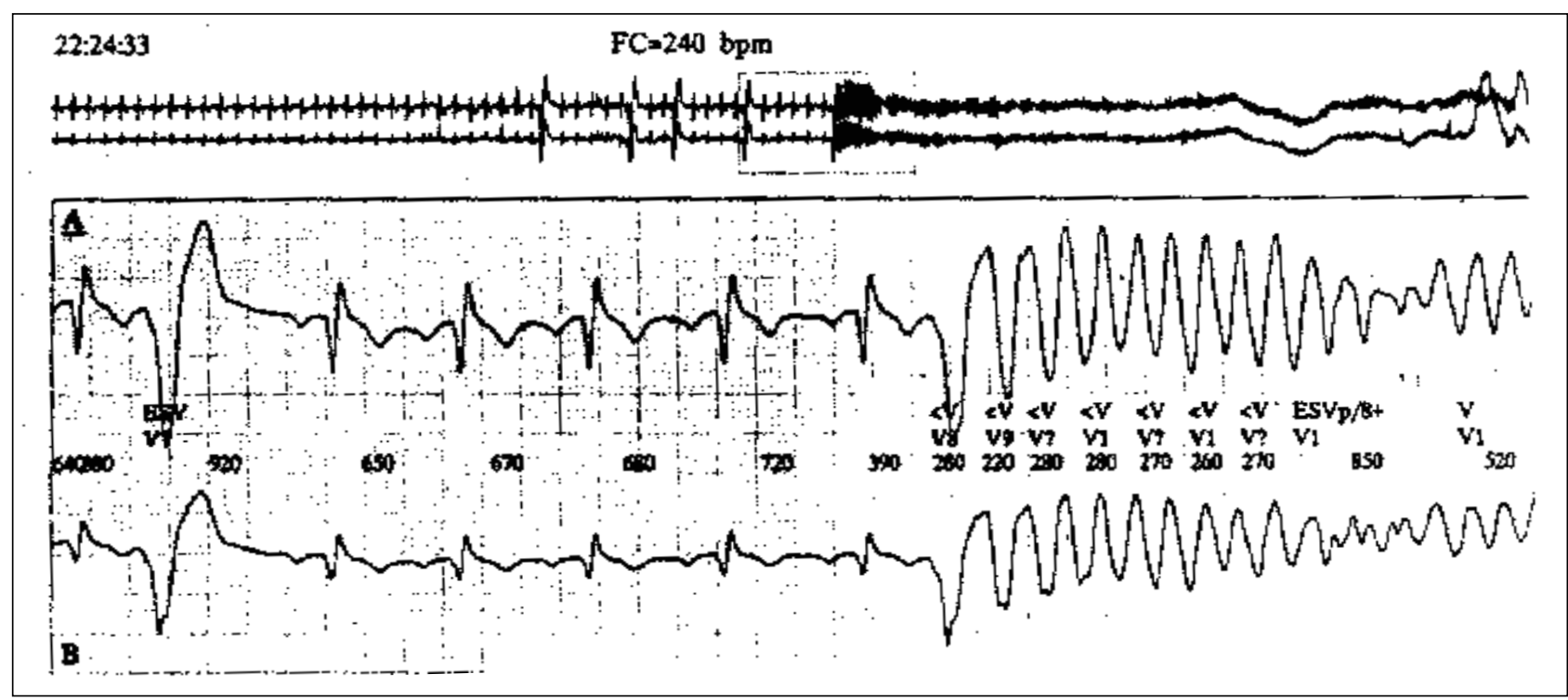

Fig. 2 - Registro de Holter documentando a morte súbita de portador de displasia arritmogênica do ventrículo direito (extraído da ref. 5).

de infiltração ou redistribuição da vasculatura pulmonar. $\mathrm{O}$ tamanho da silhueta cardíaca varia diretamente com o grau de dilatação das câmaras direitas ${ }^{14}$.

Angiocintigrafia cardíaca isotópica - Fornece dados sobre a contratilidade global e segmentar dos dois ventrículos ${ }^{32}$.

Ressonância magnética - Este método permite uma análise morfológica dos dois ventrículos e sua atividade contrátil, pondo em evidência a presença de tecido adiposo, ao nível da parede ventricular, que se apresenta como sinal de brilho anormal de alta intensidade comparável com a gordura subcutânea e pericárdio ${ }^{33}$. O valor diagnóstico da RM foi avaliado em 36 pacientes com diagnóstico confirmado de DAVD, através de biópsia miocárdica, tendo sido sugerido que essa técnica pudesse ser utilizada em substituição à angiografia e possivelmente, à biópsia ${ }^{34}$. Midiri e col propuseram o diagnóstico de DAVD baseado na presença de áreas de alta densidade, indicando substituição gordurosa miocárdica, ectásia da via de saída do VD, imagens discinéticas, dilatação ventricular direita e crescimento atrial direito. Foram consideradas de alta probabilidade para DAVD, os pacientes que apresentassem pelo menos três critérios, moderada os com dois critérios e diagnóstico duvidoso os com um ou nenhum critério. Os autores consideraram que esta forma de classificação poderia ser útil no diagnóstico da DAVD $^{35}$. Julgamos que a RM colabore no diagnóstico daqueles casos em que a DAVD encontra-se mais avançada e com acometimento mais difuso e intenso ventricular direito.

Angiografia ventricular - Os critérios mais característicos são a dilatação, as anomalias da contração global e segmentar e a presença de saculações do VD ${ }^{36}$ (fig. 5). O infundíbulo pulmonar, o ápex e a parede póstero-diafragmática do VD são os sítios de predileção das dilatações, às vezes aneurismáticas, encontradas na DAVD. Este tipo de apresentação recebeu a denominação de "triângulo da displasia". Uma alteração da contratilidade de VE pode ser observada na porção inferior do septo interventricular (SIV) ${ }^{37}$.

Estudo eletrofisiológico - Este estudo permite a identificação, no endocárdio, de zonas com características patológicas, onde se identificam potenciais tardios em ritmo sinusal com características de alta freqüência e baixa amplitude (<1Mv). A estimulação ventricular programada (EVP) permite indução e término de TV monomórfica com características morfológicas e de freqüência compatíveis com às registradas espontaneamente. Nos casos de difícil indução da TV clínica, pode-se utilizar o isoproterenol ${ }^{38}$. A estimulação programada pode também ser utilizada para avaliar a possibilidade de indução de TV em casos assintomáticos, mas com significância questionada ${ }^{39}$. Em alguns casos, a EVP pode induzir fibrilação ventricular. A exploração eletrofisiológica em curso de TV é fundamental para determinar o sítio da aplicação de método ablativo. Um retardo estímulo-resposta ventricular, após uma linha isoelétrica $>100 \mathrm{~ms}$, a exemplo da TV no paciente isquêmico, pode ser evidenciado neste grupo de pacientes. Estas características, somadas ao registro de potenciais tardios e da possibilidade de resultado efetivo, a longo-prazo, com terapêutica cirúrgica por técnica de ventriculotomia simples e, em casos selecionados, através de ablação por cateter ao nível da zona "crítica", sugerem que o mecanismo de reentrada esteja diretamente envolvido na gênese da TV da DAVD.

O amplo espectro de apresentação da DAVD pode interferir na valorização dos dados obtidos através de resultados de exames complementares e da análise da história clínica. Objetivando otimizar os critérios diagnósticos da DAVD, recentemente a Sociedade Européia de Cardiologia propôs que o diagnóstico seja baseado na presença de critérios maiores e menores. Esses critérios incluem a presença de alterações estruturais globais e segmentares do VD, alterações histológicas, eletrocardiográficas, presença de 


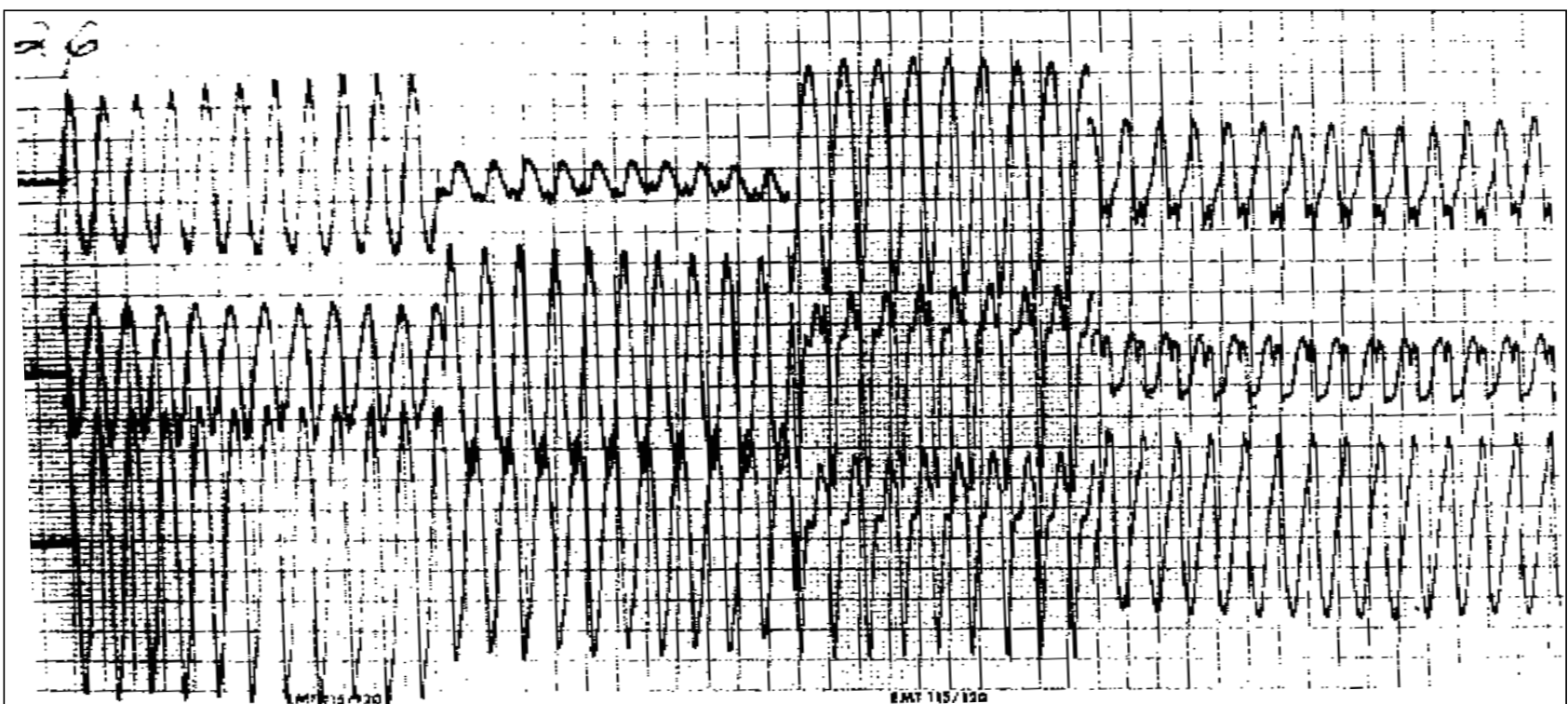

Fig. 3 - Eletrocardiograma de 12 derivações de taquicardia ventricular em portador de displasia arritmogênica do ventrículo direito. Morfologia do tipo bloqueio de ramo esquerdo e eixo elétrico superior no plano frontal.

arritmias e fatores genéticos. Baseando-se nessa classificação, o diagnóstico da DAVD existiria quando da presença de dois critérios maiores ou um critério maior associado a dois critérios menores de grupos distintos ou ainda quatro critérios menores (quadro I) ${ }^{40}$.

\section{Patogênese}

A existência de formas familiares levou à pesquisa de um possível mecanismo genético. A doença poderia, então, ser conseqüência de um distúrbio do desenvolvimento do miocárdio, já presente no embrião, secundária a algum fator genético. A apresentação clínica sugere ocorrência familiar autossômica dominante, penetração variável e expressão fenotípica polimórfica. Um defeito genético foi recentemente mapeado no cromossoma 14q 23-q24 em uma família ${ }^{41}$. Este enfoque aproxima muito a DAVD da anomalia de Uhl ${ }^{15}$.

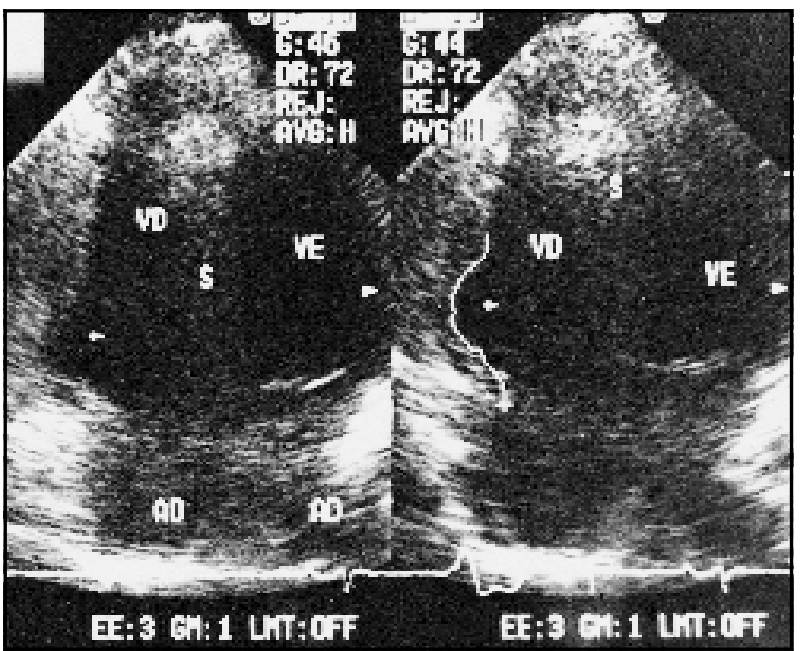

Fig. 4 - Pequena expansão aneurismática em região lateral do ventrículo direito próximo do anel da válvula tricúspide - corte apical 4 câmaras.
Segundo uma outra teoria, a DAVD seria conseqüência de uma transformação adquirida dos cardiomiócitos do VD, sob influência de um fenômeno inflamatório ou infeccioso prévio (miocardite, pericardite). Nesta teoria, a hipótese de uma infecção viral é a mais plausível, agravada ou não por uma reação auto-imune. Esta situação poderia, então, ser conseqüência de uma suscetibilidade genética. Esta última hipótese leva a dificuldades para realização de diagnóstico diferencial com a forma "fibrosa" de certas cardiomiopatias idiopáticas dilatadas ${ }^{42}$. Uma patogênese miocardítica poderia explicar o comprometimento do ventrículo esquerdo(VE) e a existência de distúrbio do ritmo atrial. Uma composição entre as duas teorias também se mostra possível. Um marcador miocárdico, geneticamente determinado, pode se mostrar especialmente sensível a um agente infeccioso, podendo-se, neste caso, evidenciar presença de sinais de displasia e de miocardite ${ }^{43}$.

Em estudo recente, Rossi procurou traçar um paralelo entre a DAVD e a cardiomiopatia chagásica, findando por sugerir a possibilidade de mecanismos patogênicos em comum, com possível participação de fatores auto-imunes/inflamatórios e microvasculares e com associação de disfunção do sistema nervoso autonômico ${ }^{44}$.

\section{Aspectos anatômicos e histológicos}

O exame anatomopatológico permite compreender a DAVD, fornecendo condições para interpretação dos dados obtidos pelo estudo eletrofisiológico. Na avaliação macroscópica, já se evidencia a existência de dilatação de VD e presença de tecido gorduroso em concentração aumentada epicárdica e médio-mural ${ }^{45}$. Basso e col identificaram aneurisma do VDem 50\%, envolvimento do VEem 47\% e do SIV em $20 \%$ de sua casuística ${ }^{46}$. A descrição completa das lesões foram realizadas durante biópsias obtidas peroperatoriamente ou post-mortem. Isto é importante, por- 


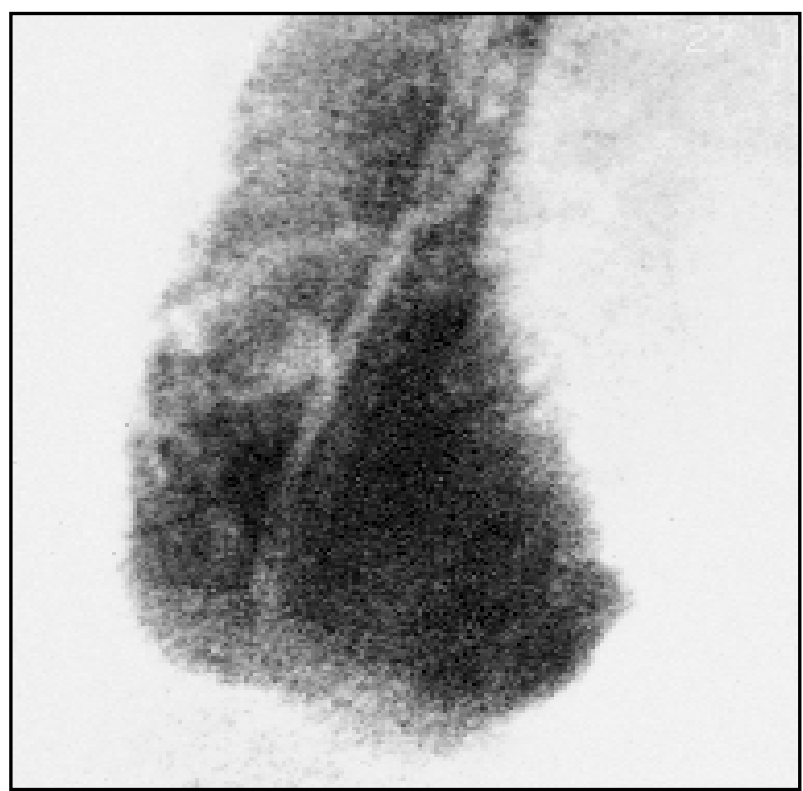

Fig. 5 - Angiografia ventricular direita em incidência oblíqua anterior direita apresentando expansão aneurismática em região septal inferior.

que, para coleta de amostras, deve-se levar em conta a topografia particular das lesões. A biópsia endomiocárdica por cateter, se feita sem atentar para este detalhe, pode demonstrar tecido normal além do fato que a confirmação diagnóstica da DAVD por biópsia, carece em sensibilidade, devido às alterações patológicas típicas estarem presentes em parede livre do VD e não no SIV, sítio habitual da biópsia endomiocárdica. Outro aspecto importante é a evidência de placas fibrosas de ordem de 1 a $2 \mathrm{~cm}$ de largura, sem relação com as zonas displásicas. Torna-se assim, muitas vezes difícil estabelecer-se o diagnóstico histológico da DAVD por biópsia endomiocárdica por cateter, mas não deixa de ser factível ${ }^{47}$. $\mathrm{O}$ aspecto que mais chama atenção é a "miogordura" coexistente no do tecido gorduroso subepicárdico e médio-mural. Os cardiomiócitos são anormais ou entrecortados por finos feixes de fibrose hialina distribuídos em intensidade decrescente da região subendocárdica para subepicárdica (fig. 6). A presença de

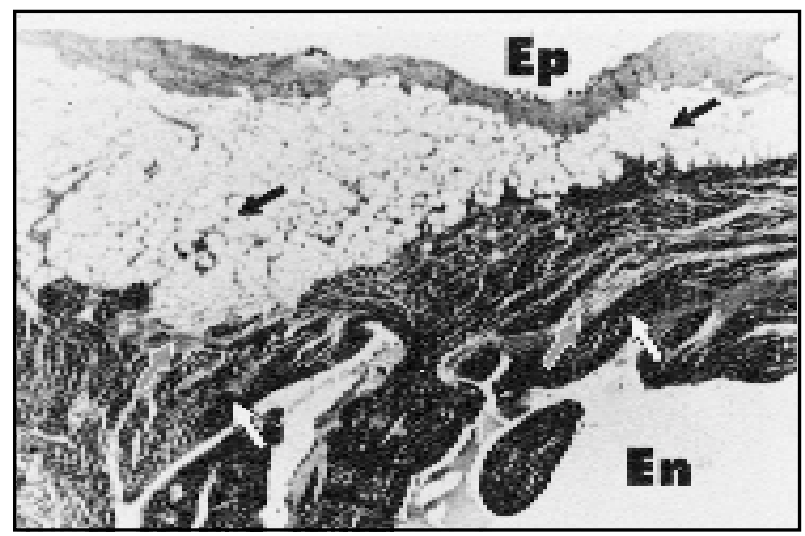

Fig. 6 - Aspecto histológico da parede livre do ventrículo direito. Presença de infiltrado adiposo de predomínio epicárdico e com envolvimento médio-mural (setas pretas). Traves de tecido fibrótico (setas cinzas) entrecortando tecido endocárdico normal (setas brancas). Ep- epicárdio; En- endocárdio.

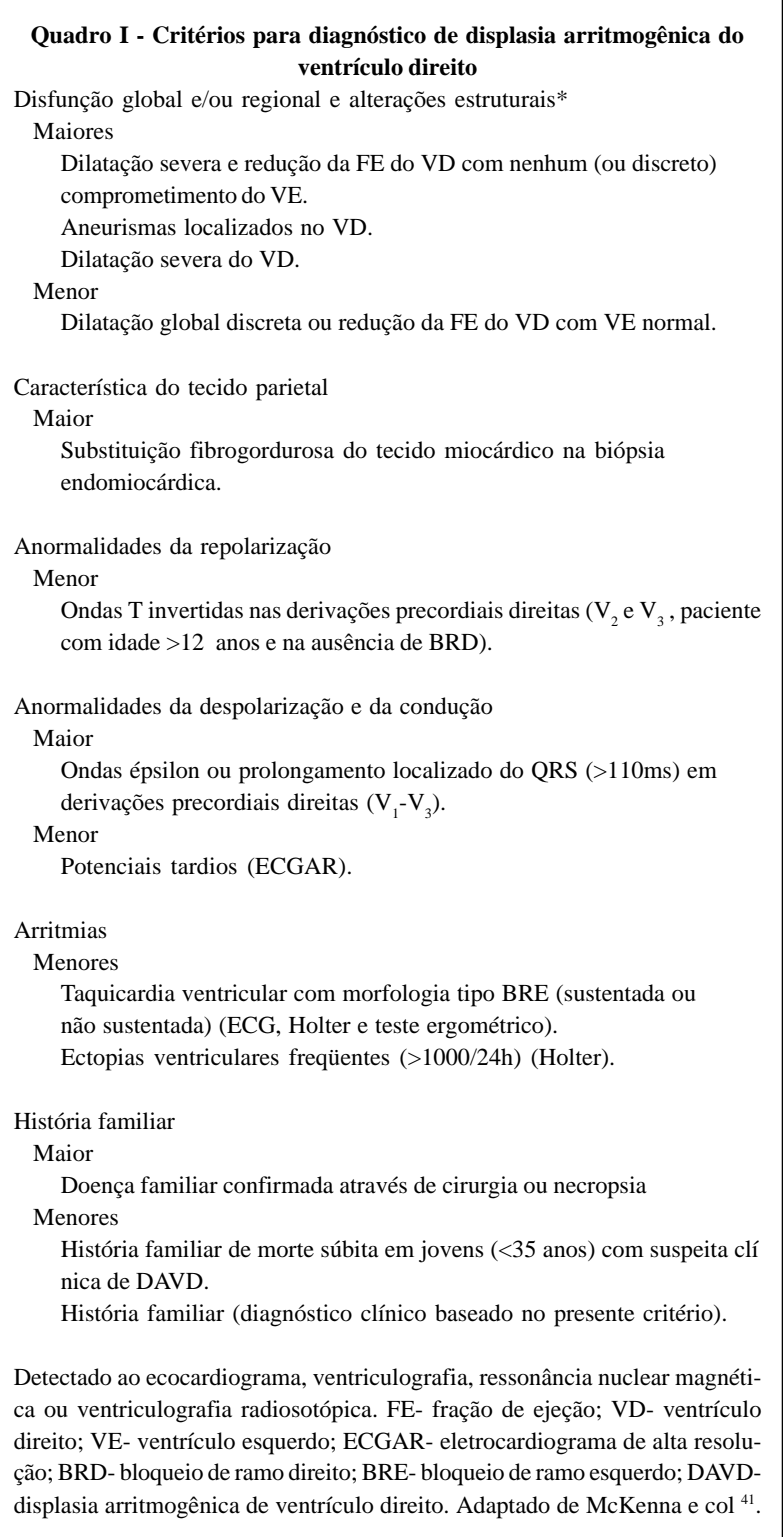

traves fibróticas é forte indicador diagnóstico de DAVD e explica por que, originalmente, considera-se que o mecanismo da displasia é resultado de um tipo específico de degeneração, quando comparado a um processo de transformação em tecido adiposo ${ }^{48}$. A degeneração gordurosa dos leiomiócitos pode levar a obstrução vascular coronária de grau variável. Em número significativo de casos, demonstrase a presença de grumos de infiltrado linfocitário dentro do tecido fibroso ${ }^{49}$. Este aspecto é então diferente de uma sobrecarga de tecido adiposo, compondo a região do epicárdio de um VD normal no qual o aspecto de fibrose não se mostra presente ${ }^{48}$. Recentemente, Basso e col propuseram, de acordo com o substrato histopatológico, a subdivisão da DAVD em dois tipos: forma fibrogordurosa e forma gordurosa. De acordo com esses autores, a variedade fibrogordurosa mostrou uma diferença significativa em termos de espessura da parede do VD $(2,9 \pm 1,0$ contra $4,3 \pm 1,2 \mathrm{~mm}, \mathrm{P}<0,0001)$, ocorrência de aneurisma do $\mathrm{VD}(78 \%$ 


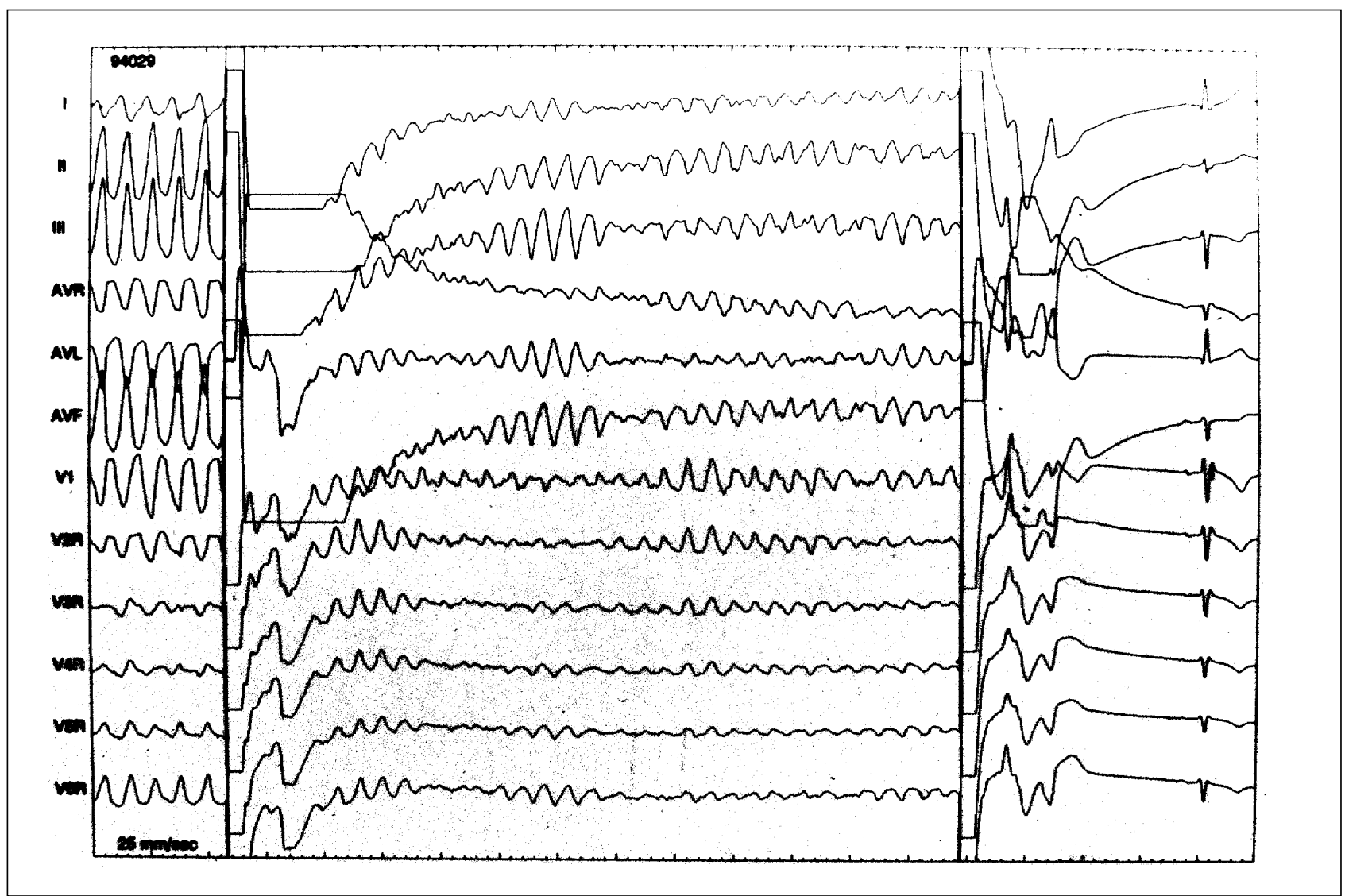

Fig. 7- Reversão de taquicardia ventricular de via de saída do ventrículo direito através de cardioversor-desfibrilador implantável. Primeiro choque induzindo fibrilação ventricular. Após $2^{\circ}$ choque reversão para ritmo sinusal. Observar características eletrocardiográficas típicas de displasia arritmogênica do ventrículo direito em ritmo sinusal

contra $8 \%, \mathrm{P}<0,001)$ e miocardite focal $(100 \%$ contra $17 \%$, $\mathrm{P}<0,001)$. O envolvimento do VEe SIV foi exclusivo da variedade fibrogordurosa. Esta descrição também não está de acordo com os achados histológicos atribuídos à anomalia de Uhl, na qual evidencia-se um desaparecimento total de cardiomiócitos na parede livre de VD, levando a um acolamento do epicárdio ao endocárdio, de forma localizada ou difusa ${ }^{50}$. O VD se mostra, então, extremamente dilatado, sendo descrito como de aspecto "papiráceo", que se opõe a um VE normal. A diferenciação entre estas duas doenças traz consigo importantes implicações clínico-cirúrgicas. As alterações ultra-estruturais mais evidentes são concernentes aos discos intercalares. Em estudo recente, James e col sugeriram que a morte celular miocárdica na DAVD possa representar uma "morte programada" denominada apoptose. Da mesma forma que a involução do miocárdio do VD resulta de um remodelamento normal pós-natal, mais provavelmente mediado por apoptose, na DAVD a apoptose, na forma recorrente ou contínua, pode levar a desaparecimento miocárdico progressivo acompanhado por substituição fibrogordurosa ${ }^{51}$. Esta perda progressiva miocárdica, com substituição peculiar por fibrogordura ou gordura, entremeadas por ilhas de miofibrilas parcialmente degeneradas podem formar, em determinadas regiões, uma estrutura de conformação complexa, podendo assim justificar a característica de anisotropismo anormal e condução lenta.

\section{Diagnóstico diferencial}

As arritmias cardíacas podem ser o primeiro sinal de cardiomiopatia idiopática antes da dilatação das câmaras cardíacas. Quando identificadas como sendo de origem em câmaras direitas, estas arritmias podem levar a um falso diagnóstico de DAVD. Já o paciente com DAVD pode apresentar dilatação das câmaras cardíacas associada à arritmia ventricular. Crê-se que a avaliação da morfologia e da função do VD, feita de forma seriada, a longo prazo, pode ser um marcador de diferenciação desses pacientes.

A TV idiopática de origem de via de saída de VD pode representar, devido às suas características morfológicas similares às da TV da DAVD e de origem em mesma localização, um diagnóstico diferencial com as formas "frustras" de DAVD. Porém, nesse subgrupo, as TVs são dificilmente indutíveis, não se evidenciando alterações no ecocardiograma e RM, o ECGAR é geralmente negativo e pode apresentar cura espontânea. Em estudo recente, Grimm e col avaliaram através de RM e ECGAR portadores de TV com padrão morfológico de via de saída do VD, ECG de 12 derivações e ecocardiograma normais quanto à possibilidade de se tratar de formas localizadas de DAVD, e concluíram que os achados da RM e ECGAR normais não favorecem a hipótese de que formas localizadas de DAVD sejam o substrato arritmogênico na maioria dos portadores de TV idiopática 
de via de saída do VD ${ }^{52}$. Outro dado que deve ser levado em consideração, favorecendo o diagnóstico de DAVD, é a presença de mais de uma morfologia de $\mathrm{TV}$, principalmente quando o eixo da TV no plano frontal for normal ou para esquerda. A anomalia de Uhl, em sua apresentação típica, muito rara, que compromete principalmente recém-natos, apresenta uma característica histopatológica bem diferenciada em relação à displasia. Além disso, esta doença é geralmente fatal, evoluindo com falência cardíaca independente da presença de TV. Já as formas localizadas da anomalia de Uhl, com raros casos relatados na literatura, podem apresentar uma evolução menos florida.

\section{Tratamento}

Os antiarrítmicos, na forma de monoterapia ou em associação, receitados quase sempre de forma empírica, têm sido a abordagem terapêutica mais comumente usada.

A amiodarona e, mais recentemente, o sotalol ${ }^{53}$ constituíram-se as drogas de escolha, isoladas ou associadas a drogas da classe IA ou IC. Entretanto, não existem estudos prospectivos que tenham demonstrado benefício sobre a mortalidade e incidência de efeitos adversos com esta terapêutica.

Nos pacientes mais jovens, pode-se discutir um tratamento mais radical devido aos riscos inerentes e à variabilidade de aderência ao tratamento a longo prazo, ou intolerância às drogas antiarrítmicas. $\mathrm{O}$ mesmo pode ser proposto para as formas mais rebeldes de TV. O método ablativo, por fulguraçãoe, mais recentemente, a radiofrequiência, embora com índice de sucesso geralmente não satisfatórios, podem ser utilizados como opção terapêutica em casos selecionados. Os melhores resultados através desta técnica são obtidos nos casos de TV incessante que, devido às características de frequiência e tolerabilidade das mesmas, permite um mapeamento endocavitário mais refinado ${ }^{54}$. A ablação por radiofrequiência pode também ser utilizada como terapêutica coadjuvante, nos pacientes que necessitam implante de cardioversor-desfibrilador implantável (CDI) e têm na TV incessante uma contra-indicação para o implante do CDI.
Devido à DAVD poder apresentar um caráter de doença progressiva, novas formas de TV podem surgir durante a evolução, interferindo a longo prazo nos resultados obtidos através da ablação.

Enfim, em casos ressuscitados de morte súbita, com fibrilação ventricular documentada ou flutter ventricular, sem TV indutível ou na TV refratária, oCDI ou, menos comumente, otransplante cardíaco poderá ser utilizado (fig. 7).

\section{Evolução e prognóstico}

Poucos estudos relatam uma longa evolução desta doença ${ }^{56}$. Tomando-se como base os estudos realizados pelos grupos sueco e americano, nos quais os pacientes foram tratados pelos métodos convencionais, não baseados na certeza da não indutibilidade do distúrbio do ritmo por métodos eletrofisiológicos ${ }^{57,58}$, a mortalidade observada é de $2 \%$ ao ano. Fontaine e col observaram um melhor prognóstico provavelmente relacionado à demonstração do benefício da droga antiarrítmica, usualmente amiodarona ou sotalol, através da não indutibilidade da arritmia clínica frente à estimulação programada e controle através de Holter seriado.

\section{Conclusão}

A DAVD é uma entidade anatomoclínica onde a incidência na população geral é ainda mal conhecida. Sua identificação entre os profissionais de risco se impõe. É importante que além da cardiomiopatia hipertrófica, a DAVD seja lembrada como causa importante de morte súbita em jovens e atletas. Seu mecanismo etiopatogenético, como doença genética ou adquirida, ou seqüela de miocardite, merece estudos aprofundados.

Estudos prospectivos são necessários no intuito de identificar sua real incidência na população. Sua história natural e os resultados dos tratamentos com drogas antirrítmicas, métodos ablativos, desfibrilador implantável ou transplante cardíaco devem ser avaliados.

\section{Referências}

1. Fontaine G, Guiraudon G, Frank Ret al - Stimulation studies and epicardial mapping in ventricular tachycardia: study of mechanisms and selection for surgery. In: Kulbertus HE - Reentrant Arrhythmias, ed. Lancaster: MTP Pub, 1977; 33450 .

2. Atallah G, Tabib A, Touboul Pet al - Right ventricular dysplasia as a cause of sudden cardiac death. Study of 1000 post-mortem examinations. J Am Coll Cardiol 1992; 19(suppl A): 121.

3. Lobo FV, Heiggtveit HA, Butany J, Silver MD, Edwards JE - Right ventricular dysplasia: Morphological findings in 13 cases. Can J Cardiol 1992; 8: 261-8.

4. Thiene G, Nava A, Corrado D, Rossi L, Pennelli N-Right ventricular cardiomyopathy and sudden death in young people. N Engl Med 1988; 318: 129-33.

5. Aouate P, Fontaliran F, Fontaine Getal-Holter et mort subite. Interêt dans un cas de dysplasie ventriculaire droite arythmogéne. Arch Mal Coeur 1993; 86: 363-7.

6. Fontaine G, Guedon-Moreau L, Frank Ret al - La displasie ventriculaire droite. Ann Cardiol Angeiol 1992; 41: 399-410.

7. Vermani R, Robinowitz M, Clark MA, McAllister HA - Sudden death and partial absence of the right ventricular myocardium. Arch Pathol Lab Med 1982; 106: 163-7.
8. Thiene G, Gambino A, Corrado D, Nava A - The pathological spectrum underlying sudden death in athletes. New Trends Arrhythmias 1985; 3: 323-31.

9. Fontaine G, Fontaliran F, FrankR, Chomette G, Grosgogeat $Y$ - Les causes de mort subite chez les sportifs. Arch Mal Coeur 1989; 82: 107-11.

10. Slama R, Leclercq JF, Coumel Ph-Paroxysmal ventricular tachycardia in patients with apparently normal hearts. In: Zipes DP, Jalife J, eds - Cardiac Electrophysiology and Arrhythmias. Orlando: Grune \& Statton, 1985: 545-52.

11. Nava A, Thiene G, Canciani B et al - Familial ocurrence of right ventricular dysplasia: a study involving nine families. J Am Coll Cardiol 1988; 12: 1222-8.

12. Thiene G, Nava A, Corrado D et al - Right ventricular cardiomyopathy and sudden death in young people. N Engl J Med 1988; 318: 129-33.

13. Blomstrom-Lundqvist C, Enestrom S, Edvardsson N, Olsson SB Arrhythmogenic right ventricular dysplasia presenting with ventricular tachycardia in a father and a son. Clin Cardiol 1987; 10: 277-83.

14. Marcus FI, Fontaine G, Frank R et al - Right ventricular dysplasia: a report of 24 cases. Circulation 1982; 65: 384-99.

15. Fontaine G, Umemura J, Di Donna Pet al - La duree des complexes QRS dans la dysplasie ventriculaire droite arythmogène. Un nouveau marqueur 
diagnostique non invasif. Ann Cardiol Angéiol 1993; 42: 399-405.

16. Marcus F, Fontaine G - Arrhythmogenic right ventricular dysplasia/ cardiomiopathy: review. PACE 1995; 18: 1298-314.

17. Bharati S, Ciraulo DA, Bilitch M, Rosen KM, Lev M-Inexcitable right ventricle and bilateral bundle branch block in Uhl's disease. Circulation 1978; 57: 63644.

18. Tonet JL, Castro-Miranda R, Iwa T, Poulain F, Frank R, Fontaine G-Frequency of supraventricular tachyarhythmias in arrhythmogenic right ventricular dysplasia. Am J Cardiol 1991; 67: 1153.

19. Coumel P, Leenhardt A, Haddad G - Exercise ECG: Prognostic implications of exercise induced arrhytmias. PACE 1994; 17: 417-27.

20. Edvardsson N, Hirsch I, Linblad A, Olsson SB - Properties of late potentials in arrhythmogenic right ventricular dysplasia. Circulation 1984; II: 373.

21. Iwa T, Nakazato Y, Lascault G, Tonet J, Frank R, Fontaine G-Signal averaged electrogram in detection of patients with non-ischemic ventricular tachycardia. Circulation 1992; 86(suppl-I): 388.

22. Leclercq JF, Denjoy I, Maison-Blanche P et al - Potentiels tardifs et dysplasie ventriculaire droite arythmogéne. Ann Cardiol Angeiol 1990; 39: 281-5.

23. Kinoshita O, Fontaine G, Elias J et al - Time and frequency-domain analyses of the signal-averaged ecg in patients with arrhythmogenic right ventricular dysplasia. Circulation 1995; 91: 715-21.

24. Fellat R, Frank R Le Guludec D - Correlation between late potentials and right ventricular radionuclide fourier phase analysis abnormalities. Eur Heart J 1993; 14: 449A.

25. Blomstrom-Lundqvist C, Olsson SB, Edvardsson N - Follow-up by repeated signal averaged QRS in patients with the syndrome of arrhythmogenic right ventriclar dysplasia. Eur Heart J 1989; 10(suppl D): 54-60.

26. Baran A, Nanda NC, Falkoff MD, Barold SS, Gallagher JJ- Two-dimensional echocardiografic detection of arrhythmogenic right ventricular dysplasia. Am Heart J 1982; 103: 1066-7.

27. Iliceto S, Izzi M, DeMartino G, Rizzon P - Echo Doppler evaluation of right ventricular dysplasia. Eur Heart J 1989; 10(suppl-D): 29-32.

28. Kisslo JA - Two-dimensional echocardiography in arrhythmogenic right ventricular dysplasia. Eur Heart J 1989; (supp-D): 22-6.

29. Morgera T, Salvi A, Albert E, Silvestri F, Camerini F - Morphological findings in apparently idiopathic ventricular tachycardia. An echocardiographic haemodynamic and histologic study. Eur Heart J 1985; 6: 323-34.

30. Robertson JH, Bardy GH, German LD, Gallagher JJ, Kisslo JA - Comparison of 2dimensional echocardiografic and angiographic findings in arrhythmogenic right ventricular dysplasia. Am J Cardiol 1985; 55: 1506-08.

31. Scognamiglio R, Fasoli G, Nava A et al - Relevance of subtle echocardiographic findings in early diagnosis of the concealed form of right ventriclar dysplasia. Eur Heart J 1989; 10(suppl D): 27-8.

32. Bruzzone F, Borziani S, Clavario P et al - Right ventricular dysplasia. Radiosotopic angiography. Eur Heart J 1989; 10(supp D): 37-41.

33. Wolf JE, Rose-Pittet L, Page E et al - Mise en evidence par l'RM des lesions pariétales au cours de dysplasies arythmogénes du ventricule droit. Arch Mal Coeur 1989; 89: 1711-17.

34. Auffermann W, Wichter T, Breithardt G - Arrhythmogenic right ventricular disease:MR imaging versus angiography. Am J Radiol 1993; 161: 549-55.

35. Midiri M, Finazzo M, Brancato M et al - Arrhythmogenic right ventricular dysplasia: MR features. Eur Radiol 1997; 7: 307-12

36. Daubert JC, Descaves C, Foulgoc JLet al - Critical analysis of cineangiographic criteria for diagnosis of arrhythmogenic right ventricular dysplasia. Am Heart J 1988; 115: 448-59.

37. Foale RA, Nihoyannopoulos P, Ribeiro PA et al - Right ventricular abnormalites in ventricular tachycardia of right ventricular origin: relation to electrophysiological abnormalities. Br Heart J 1986; 56: 45-54

38. Haissaguerre M, Le Metayer P, D'Ivernois C et al - Distinctive response of arrhythmogenic right ventricular disease to high dose of isoproterenol. Pace 1990; 13: 2119-26.

39. D.Biase M, Favale S, Assari VM, Amodio G, Chiddo A, Rizzon P- Programmed stimulation in patients with minor forms of right ventricular dysplasia. Eur Heart J 1989; 10(supp D): 49-53.

40. Rampazzo A, Nava A, Danieli GA et al - The gene for arrhythmogenic right ventricular cardiomyopathy maps to chromosome 14q-23-q24. Hum Mol Genet 1994; 3: 959-62.

41. McKenna WJ, Thiene G, Nava A et al - Diagnosis of arrhythmogenic right ventricular dysplasia/cardiomuopathy. Task Force of the Working Group Myocardial and Pericardial Disease of the European Society of Cardiology and of the Scientific Council on Cardiomyopathies og the International Society and Federation of Cardiology. Br Heart J 1994; 71: 215-8.

42. Fontaine G, Fontaliran F, Linares-Cruz E, Chomette G- The arrhythmogenic right ventricule. In: Iwa T, Fontaine G, ed - Cardiac Arrhythmias. Recent Progress in Investigation and Management. Elsevier Science Pub. The Hague 1988; 8: 189202.

43. Kawai C, Abllmann WH - Pathogeneses of myocarditis and cardiomyophaty. recent experimental and clinical studies. University of Tokio: Press Pub Tokyo, 1987.

44. Rossi M-Comparison of Chagas heart disease to arrhythmogenic right ventricular cardiomyopathy. Am Heart J 1995; 129: 626-9.

45. Kawamura O, Ohaki Y, Nakatani Y - Idiopathic right ventricular dilatation. special reference to "arrhythmogenic right ventricular dysplasia" and analogous lesions. Acta Pathol Jpn 1986; 36: 1693-705.

46. Basso C, Gaetano T, Corrado D, Angelini A, Nava A, Valente M-Arrhythmogenic right ventriclar cardiomyopathy. Dysplasia, dystrophy, or myocarditis? Circulation 1996; 94: 983-91

47. Hasumi M, Sekiguchi M, Hiroe M, Kasanuki H, Hirosawa K - Endomyocardial biopsy approach to patients with ventricular tachycardia with special reference to arrhythmogenic right ventricular dysplasia. Jpn Circ J 1987; 51: 242-9.

48. Fontaliran F, Fontaine G, Fillette F, Aouate P, Chomette G, Grosgogeat Y Frontières nosologiques de la dysplasie arythmogène. Variations quantitatives du tissu adipeux ventriculaire droit normal. Arch Mal Couer 1991; 84: 33-8.

49. Sabel KG, Blomstrom-Lundqvist C, Olsson SB - Arrhythmogenic right ventricular dysplasia in brother and sister. Is it related to myocarditis? Pediatr Cardiol 1990; 11: 113-16.

50. Uhl HS - A previously undescribed congenital malformation of the heart: Almost total absence of the myocardium of the right ventricule. Bull John Hopkins Hosp 1952; 91: 197-205.

51. James TN, Nichols MM, Sapire DW, DiPatre PL, Lopes SM - Complete heart bkick and fatak right ventricular failure in an infant. Circulation 1996; 93: 15881600 .

52. Grimm W, List-Hellwig E, Hoffmann J et al - Magnetic resonance imaging and signal-averaged electrocardiography in patients with repetitive monomorphic ventricular tachycardia and otherwise normal electrocardiogram. PACE 1997; 20: 1826-33.

53. Wichter T, Borggrefe M, Haverkamp W - Efficacy of antiarrhythmic drugs in patients with arrhythmogenic right ventricular disease. Results in patients with indutible and nonindutible ventricular tachycardia. Circulation 1992; 86: 2937.

54. Fontaine G, Frank R, Gallais Y et al - Radiofrequency catheter ablation of ventricular tachycardia. Arch Mal Coeur 1996; 89: 99-107.

55. Oteo JF, Alonso-Pulpon L, Cavero MA et al - Right ventricular arrhythmogenic dysplasia. The role of heart transplantation in its management. Rev Esp Cardiol 1994; 47: 839-42

56. Higuchi S, Caglas NM, Shumada R, Yamada A, Takeshita A, Nakamura M - 16 Year follow-up of arrhythmogenic right ventricular dysplasia Am Heart J 1984; 108: 1363-5.

57. Blomstrom-Lundqvist C, Sabel KG, Olsson SB - A long-term follow-up of 15 patients with arrhythmogenic right ventricular dysplasia. Br Heart J 1987; 58: 477-88.

58. Marcus FI, Fontaine G, FrankR, Gallagher JJ, Reitter MJ - Long-term follow-up in patients with arrhythmogenic right ventricular disease. Eur Heart J 1989; 10(suppD): 68-75. 